Mechanochr omi c I umi nescence of

1- al kanoyl ami nopyr enes adsor bed ont o cel I ul ose paper s

\begin{tabular}{|l|l|}
\hline 著者 & NAGATA Ei suke , ARA Takuya , NAKANO H deyuki \\
\hline $\begin{array}{l}\text { j our nal or } \\
\text { publ i cat i on } \mathrm{t} \text { i t l e }\end{array}$ & Dyes and Pi gment s \\
\hline vol une & 141 \\
\hline page $\mathrm{r}$ ange & $48-52$ \\
\hline year & $2017-06$ \\
\hline URL & ht t p: //hdl . handl e. net /10258/00009206 \\
\hline
\end{tabular}




\title{
Mechanochromic luminescence of 1-alkanoylaminopyrenes adsorbed onto cellulose papers
}

Eisuke Nagata, Takuya Ara, and Hideyuki Nakano*

Department of Applied Chemistry, Muroran Institute of Technology, Mizumoto-cho, Muroran, Hokkaido 050-8585, Japan

*Corresponding author. Tel: +81-143-46-5753, Fax: +81-6143-46-5701, E-mail address: nakano@mmm.muroran-it.ac.jp (H. Nakano).

\begin{abstract}
We have previously reported that crystalline samples of 1-acetylaminopyrene (AAPy), 1-octanoylaminopyrene (OAPy), and 1-stearoylaminopyrene (SAPy) exhibited mechanochromic luminescence. In the present study, we have found that these 1alkanoylaminopyrenes adsorbed onto cellulose papers also exhibited mechanochromic luminescence; however, their behaviors were essentially different from those observed for their crystalline samples. In addition, these cellulose papers were found to exhibit reversible emission color change upon alternate exposure to ethanol and water vapors. It was suggested that 1-alkanoylaminopyrene molecules were dispersed in the paper without aggregation or crystallization and that intermolecular distance of 1alkanoylaminopyrenes in the paper was reduced by either mechanical stress or exposure to ethanol vapor, resulting in increasing the sites where excimers could be formed and/or in enhancing the efficiencies of energy migration to reach the excimer sites.
\end{abstract}


Keywords: Mechanochromic luminescence; Emission Color Change; Cellulose Paper; 1-Alkanoylaminopyrene; Excimer Formation

\section{Introduction}

Photoluminescent materials whose emitting properties are switchable by controlling the environments are of interest from both viewpoints of fundamental sciences and practical applications. Among them, materials that exhibit reversible change in fluorescent color induced by mechanical stress such as grinding have recently been received attention. Such phenomena were often referred to as "mechanochromic luminescence", "mechanofluorochromism", "piezofluorochromism" and so on. In the last decade, a variety of mechanochromic luminescent materials including both organometallic and pure organic materials have been reported [1-28] and the phenomena were generally believed to be due to change in intermolecular interactions caused by altering the molecular arrangements by mechanical stress. Creation of new mechanochromic luminescent systems and elucidation of the mechanism are the subjects of interest from the viewpoints of both fundamental sciences and practical applications.

We have been investigating two series of mechanofluorochromic materials. One of them is a series of amorphous molecular materials beased on diarylaminobenzaldehyde analogues, that exhibited mechanochromic luminescence caused by the formation of the amorphous state by grinding their crystalline samples [29-32]. The other is a series of 1-alkanoylaminopyrenes including 1-acetylaminopyrene (AAPy), 1octanoylaminopyrene (OCPy) and 1-stearoylaminopyrene (SAPy) [ 33 ]. Their emission colors in the pristine crystals were found to change from bluish purple to 
yellowish green induced by mechanical grinding and to recover by immersing to ethanol. X-Ray crystal structure analysis, electronic absorption and fluorescence spectroscopies, fluorescence lifetime analysis, and powder X-ray diffraction analysis of AAPy suggested that the observed mechanofluorochromism of crystalline samples was caused by increasing of crystal defects by grinding to enhance the excimer emission.

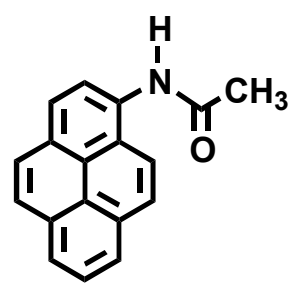

AAPy

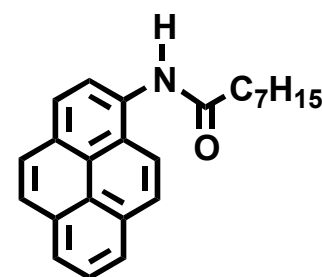

OAPy

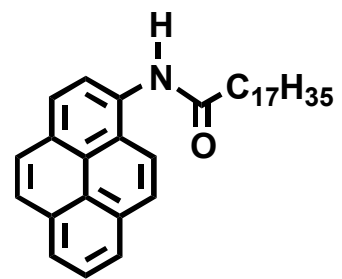

SAPy

In considering the application of such mechanochromic materials, it is thought that the form of film or paper is preferable for practical use. Thus, AAPy, OAPy, and SAPy were adsorbed onto cellulose papers and their emission properties were investigated in the present study. It was found that such cellulose papers also exhibited mechanochromic luminescence; however the behaviors were essentially different from those observed for their crystalline samples.

\section{Experimental}

AAPy, OAPy, and SAPy were prepared by the method described in our previous paper [33]. Pyrene (Py) was obtained commercially (Nacalai Tesque, Inc.) and used without further purification. Adsorption of pyrene derivatives into cellulose paper was made as follows: a cellulose paper (No. 4, Kiriyama glass Co.) with a diameter of 40 $\mathrm{mm}$ was immersed in the toluene solution of pyrene derivatives with given concentration $(\mathrm{Cp})$ for given time (Ti). After the paper was dried at ambient 
atmosphere, the resulting paper was exposed to water vapor to initialize and again dried at ambient to obtain the sample paper to investigate. Emission spectra were measured by means of FP-8300 spectrofluorometer (JASCO Co). Thickness of the paper was measured by means of a micrometer (Mitutoyo Co.). Scanning electron microscopy (SEM) was made by means of JMS-6380A (JEOL Ltd.).

\section{Results and discussion}

As described above, we have previously reported that crystalline powders of 1alkanoylaminopyrenes, AAPy, OAPy, and SAPy, exhibited mechanochromic fluorescence [33]. In the present study, we have investigated the mechanochromic fluorescent properties of these 1-alkanoylaminopyrenes adsorbed onto cellulose papers.

First of all, fluorescence emission of the AAPy-adsorbed paper prepared by immersing the cellulose paper into toluene solution of AAPy $\left(\mathrm{Cp}=6.8 \times 10^{-4} \mathrm{~mol} \mathrm{dm}^{-3}\right)$ for Ti of 0.5 min was examined. The paper before scratching, the paper was colorless under room light and purple emission was observed under the black light (365 nm) as shown in Fig. 1a. After writing a letter "N" by scratching using a spatula, the emission color was changed to yellowish green at the scratched area so that we could recognized the letter just under the black light as shown in Fig. 1b. When the resulting paper was exposed to water vapor, the emission color recovered to the original purple. Thus, the AAPy-adsorbed cellulose paper was confirmed to exhibit mechanochromic luminescence as well as the crystalline sample. Similar mechanochromic luminescence was also observed for OAPy- and SAPy-adsorbed papers prepared by the same conditions as the AAPy-adsorbed paper described above. 
(a)

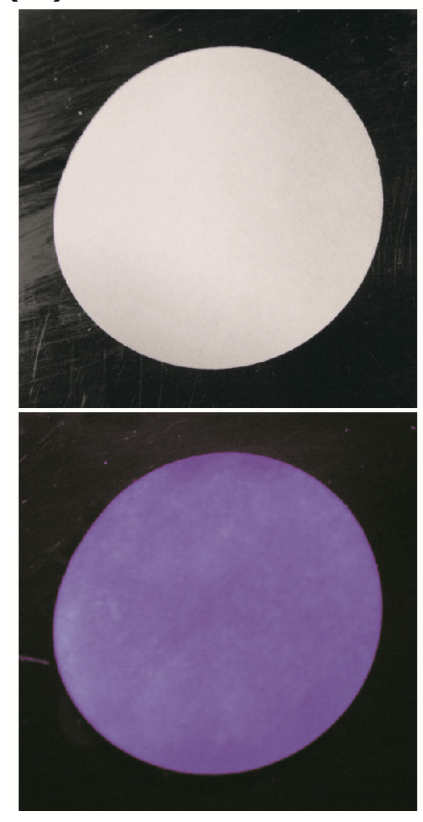

(b)

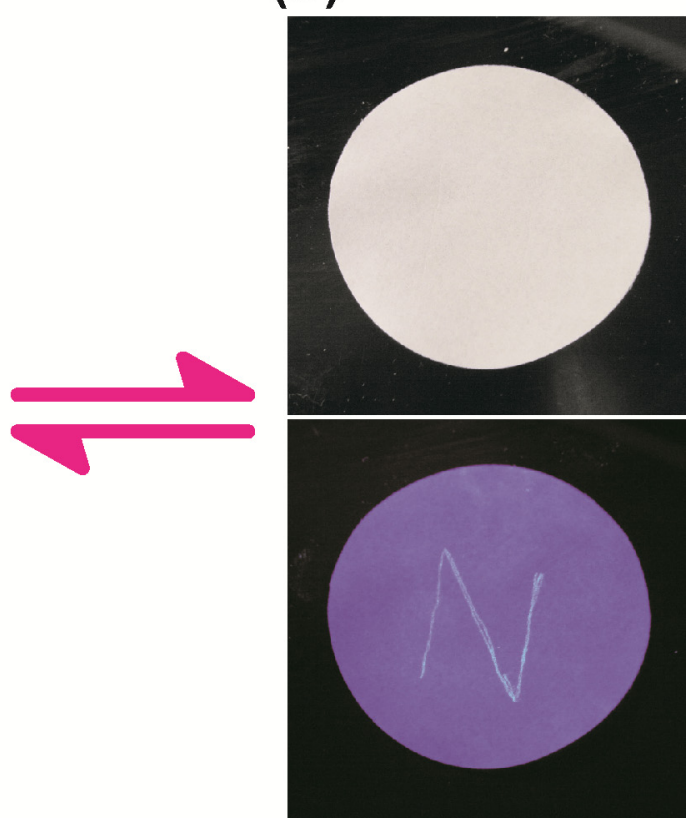

Fig. 1. Photographic images of the AAPy-adsorbed cellulose paper under room light (upper) and under the black light (365 nm) (lower).

(a) Before scratching.

(b) After writing a letter "N" by scratching with a spatula.

Then, the emission spectral change of the AAPy-adsorbed cellulose paper was examined. Before scratching, the emission in the region around $400 \mathrm{~nm}$ with vibrational structure was observed (Fig. 2a). By scratching the paper, the emission around $480 \mathrm{~nm}$ attributable to excimer emission appeared (Fig. 2b). When the resulting paper was exposed to water vapor, the spectrum was recovered to the original. Thus, it was spectroscopically confirmed that the paper exhibit mechanochromic luminescence. It is notable that the spectrum before scratching (Fig. 1a) was similar to that for ethanol solution of AAPy but different from that of recrystallized sample of AAPy (Fig. 2c). We have reported that AAPy molecules were arranged in J-aggregate manner in the crystal of AAPy, resulting in the shift of emission spectrum to longer 
wavelength relative to that for the ethanol solution of AAPy [33]. On the other hand, the present AAPy-adsorbed paper before scratching indicated the similar emission spectrum to the ethanol solution. The result suggested that AAPy molecules were dispersed in the cellulose paper without crystallization or aggregation. In addition, recovery of emission color of the scratched paper was found after exposure to water vapor whereas the emission color of ground powder of AAPy crystal could not be recovered by immersing to water. Thus, the mechanochromic fluorescence observed for AAPy-adsorbed cellulose paper in the present study was essentially different from that observed for AAPy crystal.

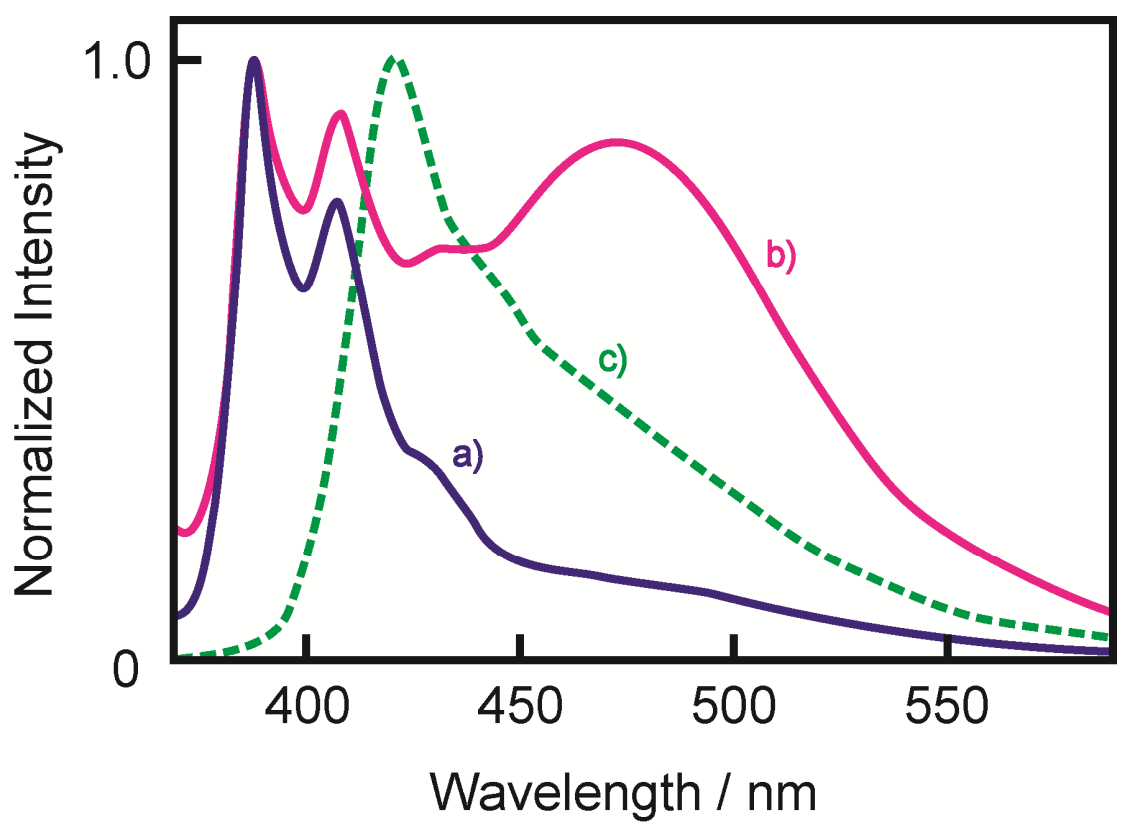

Fig. 2. Emission spectra of AAPy-adsorbed cellulose paper (a) before and (b) after scratching, together with (c) that of recrystallized solid of AAPy. $\quad \lambda_{\text {ex: }}: 350 \mathrm{~nm}$.

As reported in our previous paper [33], emission color of the ground powder of 
AAPy crystal was recovered to the original by immersing the sample into ethanol. On the other hand, the emission color of the paper after scratching could not be recovered to the original by exposure to ethanol vapor, but surprisingly the emission color of whole the paper turned to yellowish green by exposure to ethanol vapor as shown in inset of Fig. 3. The emission band around $500 \mathrm{~nm}$ attributable to excimer emission was obviously observed after exposure to ethanol vapor as shown in Fig. 3. When the resulting paper was exposed to water vapor, the emission was recovered to the original (Figs. 1a and 2a). It was confirmed that such emission color change could be reversibly induced by alternate exposure to ethanol and water vapors.

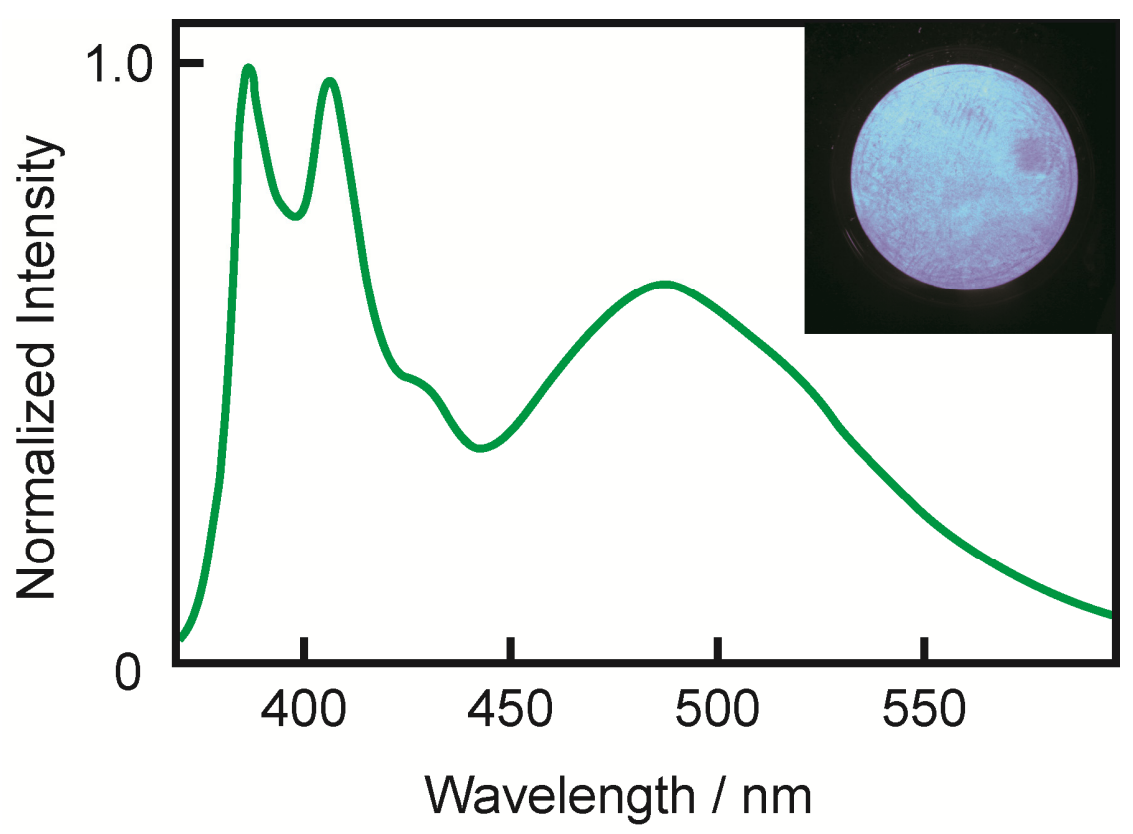

Fig. 3. Emission spectrum of AAPy-adsorbed cellulose paper after exposure to ethanol vapor $\left(\lambda_{\text {ex }}: 350 \mathrm{~nm}\right)$. Inset: Emitting image of the paper after exposure to ethanol vapor under the black light $(365 \mathrm{~nm})$. 
In order to gain information about the phenomena, the change in thickness of the paper was investigated. As shown in Fig. 4, the thickness of the paper was found to decrease after exposure to ethanol vapor and recover after exposure to water vapor. Such change in thickness was reversibly observed.

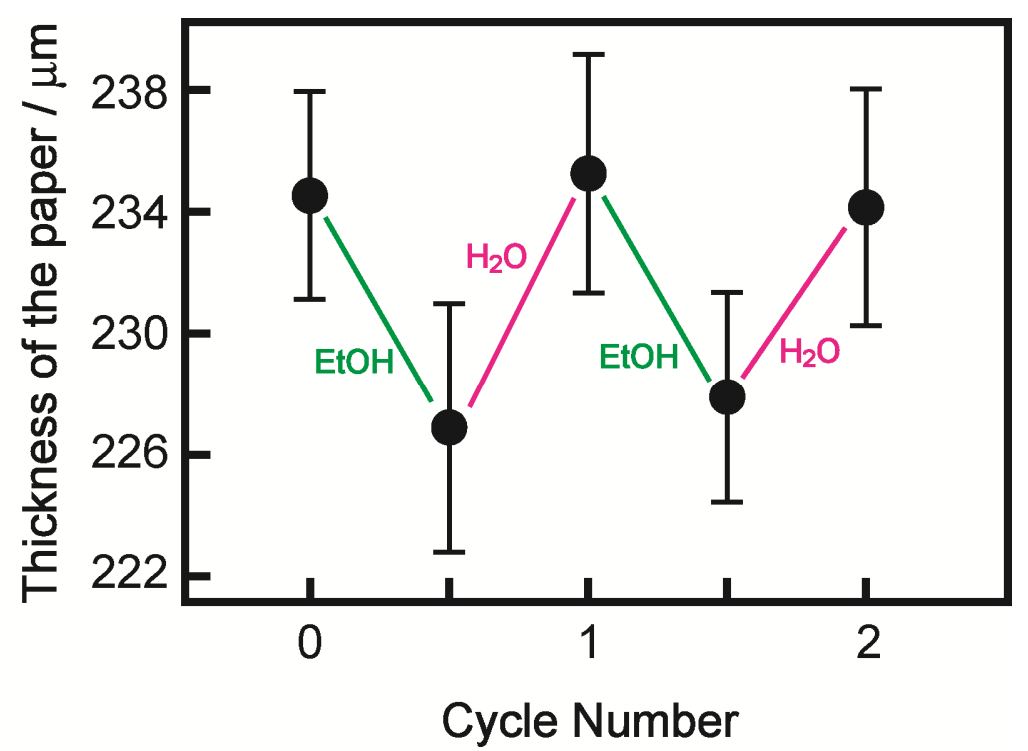

Fig. 4. Change in thickness of cellulose paper by exposure to ethanol (EtOH) and water $\left(\mathrm{H}_{2} \mathrm{O}\right)$ vapors.

Similar emission color and thickness changes of the AAPy-adsorbed cellulose paper were also found upon alternate exposure to tetrahydrofuran (THF) and water vapors. That is, when the paper was exposed to THF vapor, the emission color changed from purple to yellowish green accompanied with decreasing the thickness of the paper, and the emission color and the thickness recovered upon exposure to water vapor as shown in Figs. S1 and S2 in the supporting information.

These results suggested that the intermolecular distances play an important role for 
emission. It is conceivable that both scratching the paper and exposure to ethanol and THF vapors reduced intermolecular distances, resulting in increasing the sites where excimers could be formed and/or enhancing efficiencies of energy migration to reach excimer sites. Such effect of exposure to the ethanol vapor was also suggested by scanning electron microscopy of the cellulose papers after exposure to ethanol and water vapors. With regard to the cellulose paper after exposure to water vapor, relatively large voids were found to exist between cellulose fibers (Fig. 5a) whereas the voids seemed to become smaller after exposure to ethanol vapor (Fig. 5b). The result was consistent with the thickness change of the paper by exposure to ethanol and water vapors and suggested that the intermolecular distances of adsorbed molecules became shorter after exposure to ethanol vapor. SEM images of the cellulose paper adsorbed with AAPy after exposure to water and ethanol vapors were quite similar to those for the cellulose paper without AAPy, no aggregation of AAPy molecules being observed by adsorption or exposure to vapors. These results suggested that the effect of water and ethanol molecules to cellulose play an important role for the present emission color changes rather than that to AAPy molecules.

a)

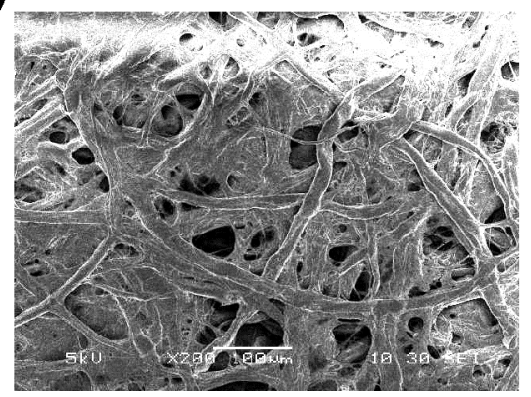

b)

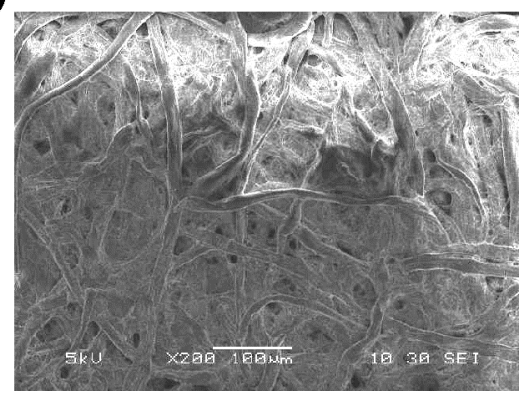

Fig. 5. SEM images of the cellulose paper after exposure to (a) water vapor and (b) ethanol vapor. 
In addition, dependence of mechanochromic fluorescence behaviors of AAPyadsorbed cellulose paper upon preparation conditions ( $\mathrm{Cp}$ and $\mathrm{Ti}$ ) were examined. All the initial sample papers as prepared exhibited purple emission like as shown in Fig. 1a; however, mechanochromic fluorescence abilities were found to depend upon the preparation conditions. Table 1 summarizes the mechanochromic fluorescence ability of AAPy-adsorbed cellulose paper depending upon the concentration $\mathrm{Cp}$ and immersing time Ti. The results indicated that higher $\mathrm{Cp}$ and longer Ti are preferable as preparing conditions to obtain mechanochromic fluorescent papers. Since the amount of adsorbed molecules was conceivable to increase with the increases in $\mathrm{Cp}$ and $\mathrm{Ti}$, the results suggested that the papers prepared with relatively high $\mathrm{Cp}$ and long Ti exhibited mechanochromic fluorescence due to appropriated intermolecular distances in the paper whereas the papers prepared with relatively low $\mathrm{Cp}$ and short $\mathrm{Ti}$ exhibited just monomeric purple emission without mechanochromic behavior by scratching due to too long intermolecular distances for excimer formation and/or for efficient energy migration. In this consideration, the paper prepared with considerably high $\mathrm{Cp}$ with enough long $\mathrm{Ti}$ was expected to exhibit excimer emission as prepared; however, we could not obtain such paper due to limited solubility of AAPy into toluene. 
Table 1. Mechanochromic fluorescence abilities of AAPy-adsorbed cellulose papers prepared by different conditions.

\begin{tabular}{cccc}
\hline $\mathrm{Cp} / \mathrm{mol} \mathrm{dm}^{-3}$ & \multicolumn{3}{c}{$\mathrm{Ti} / \mathrm{min}$} \\
\cline { 2 - 4 } & 0.5 & 5 & 10 \\
\hline $6.8 \times 10^{-7}$ & $\mathrm{n}$ & $\mathrm{n}$ & $\mathrm{n}$ \\
$6.8 \times 10^{-6}$ & $\mathrm{n}$ & $\mathrm{f}$ & $\mathrm{f}$ \\
$6.8 \times 10^{-5}$ & $\mathrm{n}$ & $\mathrm{f}$ & $\mathrm{g}$ \\
$6.8 \times 10^{-4}$ & $\mathrm{~g}$ & $\mathrm{~g}$ & $\mathrm{~g}$ \\
\hline
\end{tabular}

n: No mechanochromic fluorescence could be observed. f: Slight mechanochromic fluorescence could be observed when scratching the paper with sufficient strength. $g$ : Obvious mechanochromic fluorescence could be observed.

Since it was suspected that any fluorescent molecules with excimer-forming ability provide similar mechanochromic fluorescent paper, the papers adsorbed with other 1alkanoylaminopyrenes, OAPy and SAPy, together with unsubstituted pyrene (Py) were prepared under the condition of a variety of $\mathrm{Cp}$ for $\mathrm{Ti}$ of $0.5 \mathrm{~min}$, and their mechanochromic fluorescent behaviors were examined. The results were summarized in Table 2. We could obtain mechanochromic fluorescent papers for AAPy, OAPy and SAPy with relatively high $\mathrm{Cp}$; however, the Py-adsorbed papers prepared under the present conditions exhibited just monomeric emission without emission color change by scratching. It is conceivable that interaction between Py molecules and cellulose was relatively small so that the amount of adsorbed Py was too small to form excimer by scratching. Thus, amido groups of AAPy, OAPy and SAPy were suggested to play an 
important role for adsorbing to the cellulose paper.

Table 2. Mechanochromic fluorescence abilities of cellulose papers adsorbed with pyrene derivatives prepared under different $\mathrm{Cp}$ with Ti of $0.5 \mathrm{~min}$.

\begin{tabular}{ccccc}
\hline $\mathrm{Cp} / \mathrm{mol} \mathrm{dm}^{-3}$ & Py & AAPy & OAPy & SAPy \\
\hline $6.8 \times 10^{-7}$ & $\mathrm{n}$ & $\mathrm{n}$ & $\mathrm{n}$ & $\mathrm{n}$ \\
$6.8 \times 10^{-6}$ & $\mathrm{n}$ & $\mathrm{n}$ & $\mathrm{n}$ & $\mathrm{n}$ \\
$6.8 \times 10^{-5}$ & $\mathrm{n}$ & $\mathrm{n}$ & $\mathrm{g}$ & $\mathrm{g}$ \\
$6.8 \times 10^{-4}$ & $\mathrm{n}$ & $\mathrm{g}$ & $\mathrm{g}$ & $\mathrm{g}$ \\
\hline
\end{tabular}

n: No mechanochromic fluorescence could be observed. g: Obvious mechanochromic fluorescence could be observed.

\section{Conclusion}

AAPy-, OAPy-, and SAPy-adsorbed cellulose papers prepared by appropriate conditions were found to exhibit mechanochromic luminescence, being essentially different from those observed for their crystalline samples. These papers were also found to exhibit reversible emission color changes by alternate exposure to ethanol and water vapors. It was suggested that the emission color change was due to change in intermolecular distances by scratching and exposure to vapor. The present study suggested that the molecules with both excimer emitting ability and adsorbing ability to cellulose were promising to provide the mechanochrmic fluorescent cellulose papers. 


\section{Acknowledgement}

This work was supported by JSPS KAKENHI Grant Number JP26107006 in Scientific Research on Innovative Areas "Photosynergetics".

\section{References}

[1] Assefa Z, Omary MA, McBurnett BG, Mohamed AA, Patterson HH, Staples RJ, Fackler Jr. JP, Syntheses, Structure, and Photoluminescence Properties of the 1Dimensional Chain Compounds $\left[(\mathrm{TPA})_{2} \mathrm{Au}\right]\left[\mathrm{Au}(\mathrm{CN})_{2}\right]$ and (TPA)AuCl (TPA) 1,3,5-Triaza-7-phosphaadamantane), Inorg Chem 2002;41:6274-6280.

[2] Lee Y-A, Eisenberg R, Luminescence Tribochromism and Bright Emission in Gold(I) Thiouracilate Complexes, J Am Chem Soc 2003;125:7778-7779.

[3] Ariga K, Nakanishi T, Terasaka Y, Tsuji H, Sakai S, Kikuchi J, Piezoluminescence at the Air-Water Interface through Dynamic Molecular Recognition Driven by Lateral Pressure Application, Langmuir 2005;21:976-981.

[4] Mizukami S, Houjou H, Sugaya K, Koyama E, Tokuhisa H, Sasaki T, Kanesato M, Fluorescence Color Modulation by Intramolecular and Intermolecular $\pi-\pi$ Interactions in a Helical Zinc(II) Complex, Chem Mater 2005;17:50-56.

[5] Sagara Y, Mutai T, Yoshikawa I, Araki K, Material Design for Piezochromic Luminescence: Hydrogen-Bond-Directed Assemblies of a Pyrene Derivative, J Am Chem Soc 2007;129:1520-1521.

[6] Schneider J, Lee Y-A, Perez J, Brennessel WW, Flaschenriem C, Eisenberg R, Strong Intra- and Intermolecular Aurophilic Interactions in a New Series of Brilliantly Luminescent Dinuclear Cationic and Neutral $\mathrm{Au}(\mathrm{I})$ Benzimidazolethiolate Complexes, Inorg Chem 2008;47:957-968. 
[7] Ito H, Saito T, Oshima N, Kitamura N, Ishizaka S, Hinatsu Y, Wakeshima M, Kato M, Tsuge K, Sawamura M, Reversible Mechanochromic Luminescence of [( $\left.\mathrm{C}_{6} \mathrm{~F}_{5} \mathrm{Au}\right)_{2}(\mu-1,4-$ Diisocyanobenzene $\left.)\right], \mathrm{J}$ Am Chem Soc 2008;130:10044-10045.

[8] Sagara Y, Kato T, Stimuli-Responsive Luminescent Liquid Crystals: Change of Photoluminescent Colors Triggered by a Shear-Induced Phase Transition; Angew Chem Int Ed 2008;47:5175-5178.

[9] Sagara Y, Kato T, Mechanically induced luminescence changes in molecular assemblies, Nature Chem 2009;1:605-610.

[10] Balch AL, Dynamic Crystals: Visually Detected Mechanochemical Changes in the Luminescence of Gold and Other Transition-Metal Complexes, Angew Chem Int Ed 2009;48:2641-2644.

[11] Ooyama Y, Kagawa Y, Fukuoka H, Ito G, Harima Y, Mechanofluorochromism of a Series of Benzofuro[2,3-c]oxazolo[4,5-a]-carbazole-Type Fluorescent Dyes, Eur J Org Chem 2009:5321-5326.

[12] Chung JW, You Y, Huh HS, An B-K, Yoon S-J, Kim SH, Lee SW, Park SY, Shearand UV-Induced Fluorescence Switching in Stilbenic $\pi$-Dimer Crystals Powered by Reversible [2 + 2] Cycloaddition, J Am Chem Soc 2009;131:8163-8172.

[13] Zhang G, Lu J, Sabat M, Fraser CL, Polymorphism and Reversible Mechanochromic Luminescence for Solid-State Difluoroboron Avobenzone, J Am Chem Soc 2010;132:2160-2162.

[14] Yoon SJ, Chung JW, Gierschner J, Kim KS, Choi M-G, Kim D, Park SY, Multistimuli Two-Color Luminescence Swotching via Different Slip-Stacking of Highly Fluorescent Molecular Sheets, J Am Chem Soc 2010;132:13675-13683.

[15] Ronerts DRT, Holder SJ, Mechanochromi systems for the detection of stress, 
strain and deformation in polymeric materials, J Mater Chem 2011;21:8256-8268.

[16] Yamaguchi S, Yoshikawa I, Mutai T, Araki K, Solid-state luminescence of tetraphenylpyrene derivatives: mechano/vapochromic luminescence of 1,3,6,8tetra(40-carboxyphenyl)pyrene, J Mater Chem 2012;22:20065-20070.

[17] Teng MJ, Jia XR, Yang S, Chen XF, Wei Y, Reversible Tuning Luminescent Color and Emission Intensity: A Dipeptide-Based Light-Emitting Material, Adv Mater $2012 ; 24: 1255-1261$.

[18] Sagara Y, Komatsu T, Ueno T, Hanaoka K, Kato T, Nagano T, A Water-Soluble Mechanochromic Luminescent Pyrene Derivative Exhibiting Recovery of the Initial Photoluminescence Color in a High-Humidity Environment, Adv Funct Mater 2013;23:5277-5284.

[19] Wang Y, Liu W, Bu L, Li J, Zheng M, Zhang D, Sun M, Tao Y, Xue S, Yang W, Reversible piezochromic luminescence of 9,10-bis[(N-alkylcarbazol-3yl)vinyl]anthracenes and the dependence on $\mathrm{N}$-alkyl chain length, J Mater Chem C 2013;1:856-862.

[20] Nagura K, Saito S, Yusa H, Yamawaki H, Fujihisa H, Sato H, Shimoikeda Y, Yamaguchi S, Distinct Responses to Mechanical Grinding and Hydrostatic Pressure in Luminescent Chromism of Tetrathiazolylthiophene, J Am Chem Soc 2013;135:10322-10325.

[21] Liu W, Wang Y, Sun M, Zhang D, Zheng M, Yang W, Alkoxy-position effects on piezofluorochromism and aggregation-induced emission of 9,10bis(alkoxystyryl)anthracenes, Chem Commun 2013;49:6042-6044.

[22] Kondo M, Miura S, Okumoto K, Hashimoto M, Kawatsuiki N, Mechanochromic Luminescence Characteristics of Pyridine-Terminated Chromophores in the Solid 
State and in a Poly(vinyl alcohol) Matrix, Chem Asian J 2014;9:3188-3195.

[23] Kitani K, Kuwamura N, Tsukuda T, Yoshinari N, Konno T, Counteraniondependent mechanochromism of a photoluminescent platinum(II) complex with mixed terpyridine and thioglucose, Chem Commun 2014;50:13529-13532.

[24] Li W, Wang L, Zhang JP, Wang H, Bis-pyrene-based supramolecular aggregates with reversibly mechanochromic and vapochromic responsiveness, J Mater Chem C 2014;2:1887-1892.

[25] Sagara Y, Komatsu T, Ueno T, Hanaoka K, Kato T, Nagano T, Covalent Attachment of Mechanoresponsive Luminescent Micelles to Glasses and Polymers in Aqueous Conditions, J Am Chem Soc 2014;136:4273-4280.

[26] Zheng M, Zhang DT, Sun MX, Li YP, Liu TL, Xue SF, Yang WJ, Cruciform 9,10distyryl-2,6-bis(p-dialkylaminostyryl)anthracene homologues exhibiting alkyl length-tunable piezochromic luminescence and heat-recovery temperature of ground states, J Mater Chem C 2014;2:1913-1920.

[27] Xue P, Yao B, Liu X, Sun J, Gong P, Zhang Z, Qian C, Zhang Y, Lu R, Reversible mechanochromic luminescence of phenothiazine-based 10,100-bianthracene derivatives with different lengths of alkyl chains, J Mater Chem C 2015;3:10181025.

[28] Seki T, Takamatsu Y, Ito H, A Screening Approach for the Discovery of Mechanochromic Gold(I) Isocyanide Complexes with Crystal-to-Crystal Phase Transitions, J Am Chem Soc 2016;138:6252-6260.

[29] Mizuguchi K, Kageyama H, Nakano H, Mechanochromic luminescence of 4[bis(4-methylphenyl)amino]benzaldehyde, Mater Lett 2011;65:2658-2661.

[30] Mizuguchi K, Nakano H, Mechanofluorochromism of 4-[bis(9,9-dimethylfluoren- 
2-yl)amino]benzaldehyde, Dyes Pigm 2013;96:76-80.

[31] Okoshi K, Nakano H, Synthesis and Mechanofluorochromism of 4-[Bis(4methylphenyl)amino]acetophenone, J. Photopolym Sci Tech 2014;27:535-538.

[32] Manabe S, Nagata E, Nakano H, On-Off Type Mechanofluorochromism of a Novel Fluorescent Amorphous Molecular Material, N-\{4-[Bis(4methylphenyl)amino]benzylidene aniline, Rapid Commun Photosci 2014;3:38-41.

[33] Nagata E, Takeuchi S, Nakanishi T, Hasegawa Y, Mawatari Y, Nakano H, Mechanofluorochromism of 1-Alkanoylaminopyrene, ChemPhysChem 2015;16:3038-3043. 
